\title{
Generating functionals, consistency, and uniqueness in the integral equation theory of liquids
}

\author{
R. Fantoni ${ }^{1}$ and G. Pastore ${ }^{1}$
}

September 4, 2018

\begin{abstract}
We discuss and illustrate through numerical examples the relations between generating functionals, thermodynamic consistency (in particular the virial-free energy one), and uniqueness of the solution, in the integral equation theory of liquids. We propose a new approach for deriving closures automatically satisfying such characteristics. Results from a first exploration of this program are presented and discussed.
\end{abstract}

${ }^{1}$ Dipartimento di Fisica Teorica dell' Università and Istituto Nazionale di Fisica della Materia, Strada Costiera 11, 34014 Trieste, Italy; e-mail: rfantoni@ts.infn.it and pastore@ts.infn.it 


\section{Introduction}

Integral equation theories (IET) of the liquid state statistical mechanics are valuable tools for studying structural and thermodynamic properties of pairwise interacting fluid systems $[1,2]$. Many of these approximations to the exact relation between pair potential and pair correlation functions have been proposed in the last half century, starting from the pioneering works [3-5] to the most refined and modern approximations [6-10] which may approach the accuracy of computer simulation with a negligible computational cost.

The functional method in statistical mechanics [1] provides the most general and sound starting point to introduce IET as approximations of the exact functional relations and it is the classical statistical mechanics counterpart of the quantum density functional theory.

Notwithstanding the success of present IET to describe the structure of simple one component systems, considerable work is still devoted to derive improved approximations which could accurately describe the thermodynamics as well. Also applications to non simple or multicomponent systems are still subject of current studies.

Actually, the description of thermodynamics is one weak point of IET approaches: reasonable and apparently harmless approximations to the potential-correlation relations usually result in a dramatically inconsistent thermodynamics where many, if not all, among the exact sum rules derived from statistical mechanics, are violated.

The problem of thermodynamic inconsistency, i.e. the inequivalence between different routes to thermodynamics, actually plagues the IET approach to the point that the degree of inconsistency between different formulae for the same quantity is used as an intrinsic measurement of the quality of a closure.

In the past, some discussion of the thermodynamic consistency appeared in the literature. Hypernetted chain approximation (HNC) was recognized as a closure directly derivable from an approximation for the free energy functional [11], thus exhibiting consistency between the virial formula and the thermodynamic expression for the pressure. However, this limited consistency is not enough to guarantee a unique and faithful description of the phase diagram. Apart the problem of the remaining inconsistencies, the descriptions of the critical points and spinodal lines are seriously inadequate.

Extensive work on HNC [12-14] showed that in place of a true spinodal line, it is more appropriate to describe the numerical results as due to a region in the thermodynamic plane where no real solution of the integral equation exists. In particular, Belloni[12] showed that the disappearance of the solution originates from a branching point where two solutions merge, instead than from a line of diverging compressibility. Thus, we have direct evidence that HNC may have multiple solutions, at least in part of the phase diagram.

Empirical improvements on HNC have been proposed $[6,9,10]$ providing in many cases excellent results for one-component simple fluids. However, although reduced, the thermodynamic inconsistency problem remains and the multiple solution problem is completely untouched.

In this work we start an investigation of a new approach to IET directly addressing the two points of uniqueness of the solution and thermodynamic consistency. The basic idea is to constrain the search for new closures within the class of generating functionals which are strictly convex free energy functionals, thus enforcing the virial-energy consistency as well as the uniqueness of the solution.

In particular, in the present paper we try to answer the following questions: i) does at 
least one strictly convex free-energy functional of the pair correlation function exist? ii) what is the nature of the resulting spinodal line (if any), iii) what is the quality of the resulting thermodynamic and structural results? iv) does the simultaneous requirement of consistency and uniqueness automatically provide improved results?

As we will show, we have a positive answer for i), a thorough and interesting characterization for ii), some interesting indications for iii), and a partly negative answer for iv).

However, we can show that it is possible to exploit the control provided by the generating functional approach to easily generate new closures and we feel our procedure could be the basis of a more systematic approach to IET.

In section II we recall the connections between closures, generating functionals, thermodynamic consistency and uniqueness of solutions and we illustrate them in the well known case of HNC approximation. In section III we introduce two straightforward extensions of HNC intended to cure its problems. In Section IV numerical results are presented and discussed. In section $\mathrm{V}$ we show two possible improvements of the closures studied.

\section{Thermodynamic consistency and uniqueness of the solution of integral equations}

Since the work by Olivares and McQuarrie [15] it is known the general method to obtain the generating functional whose extremum with respect to variations of the direct $(c(r))$ or total $(h(r))$ correlation functions results in the closure relation, provided the Ornstein-Zernike equation is satisfied.

For example, if we have a closure of the form

$$
\rho^{2} c(r)=\Psi\{h(r), \beta \phi(r)\} \quad,
$$

where $\phi(r)$ is the pair interaction potential and $\Psi$ is an arbitrary function, the functional

$$
\begin{aligned}
Q[h(r), \beta \phi(r)]= & \frac{1}{2 \beta \rho}\left(\int \frac{d \mathbf{k}}{(2 \pi)^{3}}\{\rho h(k)-\ln [1-\rho h(k)]\}\right. \\
& \left.-\int d \mathbf{r} h(r) \int_{0}^{1} d t \Psi\{\operatorname{th}(r), \beta \phi(r)\}+\text { constant }\right),
\end{aligned}
$$

is such that the extremum condition

$$
\frac{\delta Q}{\delta h(r)}=0
$$

is equivalent to

$$
\rho^{2} h(r)=\Psi\{h(r), \beta \phi(r)\}+\rho \int h\left(\left|\mathbf{r}-\mathbf{r}^{\prime}\right|\right) \Psi\left\{h\left(r^{\prime}\right), \beta \phi\left(r^{\prime}\right)\right\} d \mathbf{r}^{\prime}
$$

Olivares and McQuarrie also showed how to find the generating functional if the closure is expressed in the form

$$
\rho^{2} h(r)=\Psi\{c(r), \beta \phi(r)\} \quad .
$$


In appendix A we discuss the extension of their method to the case of a closure written as

$$
\rho^{2} c(r)=\Psi\{\gamma(r), \beta \phi(r)\} \quad,
$$

where $\gamma(r)=h(r)-c(r)$ is the indirect correlation function. Notice that most of the modern closures correspond to this last case.

The possibility of translating the original integral equation into an extremum problem allows to get an easy control on two important characteristics of the approximation: thermodynamic consistency between energy and virial routes to the thermodynamics and uniqueness of the solution.

Indeed, once we get the generating functional $Q$, due to the approximations induced by the closure, there is no guarantee that its value at the extremum is an excess free energy. In order to be a free energy, the functional should satisfy the condition

$$
\frac{\delta Q}{\delta \phi(r)}=\frac{\rho}{2} g(r)
$$

where $g(r)=h(r)+1$ is the pair distribution function.

Even if this condition is not new, and mention to it is present in the literature [16], we discuss it in appendix B as well as its consequences on the thermodynamic consistency between the virial pressure and the density derivative of the free energy.

Another issue where the generating functional approach is useful is the problem of multiple solutions of the integral equations [12]. In particular, the analysis of the convexity properties of the generating functional is a very powerful tool $[17,18]$.

Let us illustrate this techniques in the case of HNC closure. It is well known $[11,15]$ that the $\mathrm{HNC}$ equation with closure

$$
c(r)=h(r)-\ln \left[g(r) e^{\beta \phi(r)}\right] \quad,
$$

can be derived from the variational principle

$$
\frac{\delta \mathcal{F}[h]}{\delta h(r)}=0 \quad,
$$

where

$$
\mathcal{F}[h]=\mathcal{F}_{O Z}[h]+\mathcal{F}_{H N C}[h],
$$

with

$$
\left\{\begin{array}{l}
\mathcal{F}_{O Z}[h]=\int \frac{d \mathbf{k}}{(2 \pi)^{3}}\{\rho \hat{h}(k)-\ln [1+\rho \hat{h}(k)]\}, \\
\mathcal{F}_{H N C}[h]=\rho^{2} \int d \mathbf{r}\left\{1+g(r)\left[\ln \left(g(r) e^{\beta \phi(r)}\right)-1\right]-h^{2}(r) / 2\right\} .
\end{array}\right.
$$

Let us call $\bar{h}(r)$ the extremum of $\mathcal{F}$, solution of the variational principle (2.9). It can be shown (see appendix B) that, within an additive constant, $\mathcal{F}[\bar{h}] /(2 \beta \rho)$ is the excess Helmholtz free energy per particle of the liquid. This ensure thermodynamic consistency between the 
route to the pressure going through the partial derivative of the free energy and the one going through the virial theorem (see appendix B). In addition, it allows to get a closed expression for the excess chemical potential without further approximations [19,20]. This feature is highly desirable for applications of IET to the determination of the phase diagrams.

Moreover if we can prove that $\mathcal{F}$, defined on some convex set of trial correlation functions $D_{c}$, is a strictly convex functional, then we know that if a solution to (2.9) exists, it corresponds to a minimum and is unique. A functional $\mathcal{F}$ is strictly convex if for all $y(r) \in D_{c}$ and $y(r) \neq 0$, we have

$$
A=\int y(r) \frac{\delta^{2} \mathcal{F}[h]}{\delta h(r) \delta h\left(r^{\prime}\right)} y\left(r^{\prime}\right) d \mathbf{r} d \mathbf{r}^{\prime}>0
$$

We calculate the second functional derivatives as follows

$$
\left\{\begin{array}{l}
\frac{\delta^{2} \mathcal{F}_{O Z}[h]}{\delta h(r) \delta h\left(r^{\prime}\right)}=\rho^{2} \int \frac{d \mathbf{k}}{(2 \pi)^{3}} e^{-i \mathbf{k} \cdot\left(\mathbf{r}+\mathbf{r}^{\prime}\right)} \frac{1}{[1+\rho \hat{h}(k)]^{2}} \\
\frac{\delta^{2} \mathcal{F}_{H N C}[h]}{\delta h(r) \delta h\left(r^{\prime}\right)}=\rho^{2} \delta\left(\mathbf{r}-\mathbf{r}^{\prime}\right)\left(\frac{1}{g(r)}-1\right)
\end{array}\right.
$$

Recalling that the static structure factor $S(k)=1+\rho \hat{h}(k)$, we find for $A$

$$
A / \rho^{2}=\int \frac{d \mathbf{k}}{(2 \pi)^{3}} \frac{\hat{y}^{2}(k)}{S^{2}(k)}+\int d \mathbf{r} y^{2}(r)\left(\frac{1}{g(r)}-1\right) .
$$

Now, the most interesting results would be to show the strict convexity of the HNC functional over the convex set of all the admissible pair correlation functions (all the $h(r) \geq-1$ and properly decaying to zero at large distance.

However, this is not the case for HNC. It has not been possible to show the positive definiteness of equation (2.14) and it has been shown [12] that in some region of the thermodynamic plane HNC does exhibit multiple solutions.

The best we can do is to obtain a more limited result. Calling $g_{1}=\sup g(r)\left(g_{1}>1\right.$ is the height of the first peak of the pair distribution function) and using Parseval theorem, we find

$$
A / \rho^{2}>\int \frac{d \mathbf{k}}{(2 \pi)^{3}} \hat{y}^{2}(k)\left(\frac{1}{S^{2}(k)}-1+\frac{1}{g_{1}}\right),
$$

from which we deduce that $A>0$ on the following set of functions

$$
D=\left\{h(r) \mid 0<S(k)<\sqrt{g_{1} /\left(g_{1}-1\right)} \quad \forall k\right\} .
$$

We conclude that $\mathcal{F}$ defined on any convex set of functions $D_{c} \subset D$ is strictly convex. Near the triple point we are sure we are out from such set since the first peak of the pair distribution function for the Lennard-Jones fluid is $g_{1} \simeq 3[21]$, so that $\sqrt{g_{1} /\left(g_{1}-1\right)} \simeq 1.2$. The first peak of the static structure factor is also close to 3 . Then we are not inside $D$ and the HNC approximation may have multiple solutions [12].

Instead, if we are in the weak coupling regime, the previous conditions tells us that there is a range where the branch of solutions going to the perfect gas limit is unique and quite isolated from other solutions. 


\section{Extensions of HNC}

The generating functional approach can be used in a systematic way to look for better closures. We think that this way, we can obtain a less empirical search method for improving closures.

In the following we report some preliminary analysis we have done. As a first test of our program, we have restricted our investigations to simple modifications of HNC functional. As we will discuss later, such a choice is certainly not optimal. However, we can learn enough to consider the approach worthwhile of further investigations and we feel the results are interesting in order to reveal more details about the characteristics of the solutions of the highly non linear IET.

\section{III.1 The HNC/H2 approximation}

We want to modify the HNC closure in order to have an integral equation with a generating functional which is strictly convex without having to restrict its definition domain. We choose as our modified $\mathrm{HNC}(\mathrm{HNC} / \mathrm{H} 2)$ closure [22]

$$
c(r)=h(r)-\ln [g(r)]-\beta \phi(r)-\alpha h^{2}(r),
$$

with $\alpha$ a parameter to be determined. The new closure generating functional is

$$
\mathcal{F}_{H N C / H 2}[h]=\rho^{2} \int d \mathbf{r}\left\{1+g(r)\left[\ln \left(g(r) e^{\beta \phi(r)}\right)-1\right]-h^{2}(r) / 2+\alpha h^{3}(r) / 3\right\} .
$$

Its second functional derivative with respect to $h$ is

$$
\frac{\delta^{2} \mathcal{F}_{H N C / H 2}[h]}{\delta h(r) \delta h\left(r^{\prime}\right)}=\rho^{2} \delta\left(\mathbf{r}-\mathbf{r}^{\prime}\right)\left[\frac{1}{g(r)}-1+2 \alpha h(r)\right] .
$$

Recalling that $h=g-1$ and $g(r)>0$ for all $r$, we see that for $\alpha=1 / 2$

$$
\frac{1}{g}-1+2 \alpha h=\frac{(1-g)^{2}}{g} \geq 0 \quad \forall g
$$

Then $\mathcal{F}_{H N C / H 2}$ is a convex functional and since $\mathcal{F}_{O Z}$ is unchanged and strictly convex (see appendix $\mathrm{C}$ ), their sum, the generating functional of the integral equation, is strictly convex.

Moreover $\left\{\mathcal{F}_{\mathrm{OZ}}[\bar{h}]+\mathcal{F}_{\mathrm{HNC} / \mathrm{H}_{2}}[\bar{h}]\right\} /(2 \beta \rho)$ continues to be the excess Helmholtz free energy per particle of the liquid since equation (2.7) holds (see appendix B).

We have then an integral equation which is both thermodynamically consistent (the pressure calculated from the virial theorem coincides with that one calculated from the Helmholtz free energy) and with a solution which, when it exists, is unique.

\section{III.2 The HNC/H3 approximation}

In the same spirit as in subsection III.1 we can try to add a term $h^{3}$ in the $\mathrm{HNC} / \mathrm{H} 2$ closure

$$
c(r)=h(r)-\ln [g(r)]-\beta \phi(r)-\alpha h^{2}(r)-\gamma h^{3}(r)
$$


with $\alpha$ and $\gamma$ parameters to be determined. We call this approximation HNC/H3. The closure generating functional is

$$
\begin{gathered}
\mathcal{F}_{H N C / H 3}[h]=\rho^{2} \int d \mathbf{r}\{1 \\
+g(r)\left[\ln \left(g(r) e^{\beta \phi(r)}\right)-1\right]-h^{2}(r) / 2 \\
\left.+\alpha h^{3}(r) / 3+\gamma h^{4}(r) / 4\right\}
\end{gathered}
$$

Its second functional derivative with respect to $h$ is

$$
\begin{aligned}
\frac{\delta^{2} \mathcal{F}_{H N C / H 3}[h]}{\delta h(r) \delta h\left(r^{\prime}\right)} & =\rho^{2} \delta\left(\mathbf{r}-\mathbf{r}^{\prime}\right)\left[\frac{1}{g(r)}-1+2 \alpha h(r)+3 \gamma h^{2}(r)\right] \\
& =\rho^{2} \delta\left(\mathbf{r}-\mathbf{r}^{\prime}\right) \frac{1-g(r)}{g(r)}\{1-2 \alpha g(r)+3 \gamma g(r)[1-g(r)]\} .
\end{aligned}
$$

In order to have the right hand side of this expression positive for $g>0$ the only choice we have is to set $\alpha=1 / 2$. In this way

$$
(1-g)[1-2 \alpha g+3 \gamma g(1-g)]=(1-g)^{2}(1+3 \gamma g),
$$

and we see that $\mathcal{F}_{H N C / H 3}$ is a convex functional if we additionally choose $\gamma>-1 /[3 \sup g(r)]$.

Once again $\left\{\mathcal{F}_{O Z}[\bar{h}]+\mathcal{F}_{H N C / H 3}[\bar{h}]\right\} /(2 \beta \rho)$ is the excess Helmholtz free energy per particle of the liquid and the thermodynamic consistency virial-free energy is ensured.

\section{Numerical results}

To solve numerically the OZ plus closure system of nonlinear equations we used Zerah's algorithm [23] and Fourier transforms were done using fast Fourier transform. In the code we always work with adimensional thermodynamic variables $T^{*}=1 /(\beta \epsilon), \rho^{*}=\rho \sigma^{3}$, and $P^{*}=P \sigma^{3} / \epsilon$, where $\sigma$ and $\epsilon$ are the characteristic length and characteristic energy of the system respectively. We always used 1024 grid points and a step size $\Delta r=0.025 \sigma$.

The thermodynamic quantities were calculated according to the statistical mechanics formulae for: the excess internal energy per particle

$$
U^{e x c} / N=2 \pi \rho \int_{0}^{\infty} \phi(r) g(r) r^{2} d r
$$

the excess virial pressure

$$
\beta P^{v} / \rho-1=-\frac{2}{3} \pi \beta \rho \int_{0}^{\infty} \frac{d \phi(r)}{d r} g(r) r^{3} d r \quad,
$$

the bulk modulus calculated from the compressibility equation

$$
B_{c}=\frac{\beta}{\rho \chi_{T}}=\frac{1}{S(k=0)},
$$

where $\chi_{T}$ is the isothermal compressibility, and the bulk modulus calculated from the virial equation

$$
B_{p}=\beta \frac{\partial P^{v}}{\partial \rho}
$$


For the calculation of $B_{p}$ once $g(r)$ and $c(r)$ had been calculated, Lado' s scheme for Fourier transforms [24] was used to determine $\partial \hat{g}(k) / \partial \rho$. Even if slow, this allows us to explicitly calculate and later invert the coefficients matrix of the linear system of equations which enters the calculation of $\partial \hat{g}(k) / \partial \rho$.

\section{IV.1 Inverse power potentials}

The general form of the inverse power potential is

$$
\phi(r)=\epsilon\left(\frac{\sigma}{r}\right)^{n}
$$

where $3<n<\infty$. For this class of fluids the thermodynamics depends only from the dimensionless coupling parameter

$$
z=\left(\rho \sigma^{3} / \sqrt{2}\right)(\beta \epsilon)^{3 / n}
$$

We performed our calculations on the $n=12,6,4$ fluids at the freezing point. We compared three kind of closures: the one of Rogers and Young [25] (RY) with thermodynamic consistency virial-compressibility and known to be very close to the simulation results, the hypernettedchain (HNC) closure, and the HNC/H2 described in subsection III.1. In each case we compared our data with the Monte Carlo (MC) results of Hansen and Schiff [26].

\section{IV.1.1 The inverse 12th power potential}

The freezing point for this fluid is at $z=0.813$. The RY $\alpha$ parameter to achieve thermodynamic consistency at this value of $z$ is 0.603 .

In table $\mathrm{i}$ we compare various thermodynamic quantities (the excess internal energy per particle, the excess virial pressure, the bulk moduli) obtained from the RY, the HNC, and the $\mathrm{HNC} / \mathrm{H} 2$ closures. In the MC calculation the excess virial pressure is 18.7 and the bulk modulus 72.7 .

In figure 1 we compare the $\mathrm{MC}$, the $\mathrm{HNC}$, and the $\mathrm{HNC} / \mathrm{H} 2$ results for the pair distribution function.

\section{IV.1.2 The inverse 6th power potential}

The freezing point for this fluid is at $z=1.54$. The RY $\alpha$ parameter to achieve thermodynamic consistency at this value of $z$ is 1.209 .

In table ii we compare various thermodynamic quantities (the excess internal energy per particle, the excess virial pressure, the bulk moduli) obtained from the RY, the HNC, and the $\mathrm{HNC} / \mathrm{H} 2$ closures. In the MC calculation the excess virial pressure is 38.8 and the bulk modulus 110.1.

\section{IV.1.3 The inverse 4 th power potential}

The freezing point for this fluid is at $z=3.92$. The RY $\alpha$ parameter to achieve thermodynamic consistency at this value of $z$ is 1.794 . 
In table iii we compare various thermodynamic quantities (the excess internal energy per particle, the excess virial pressure, the bulk moduli) obtained from the RY, the HNC, and the $\mathrm{HNC} / \mathrm{H} 2$ closures. In the $\mathrm{MC}$ calculation the excess virial pressure is 108.7 and the bulk modulus 156.

In figure 2 we compare the $\mathrm{MC}$, the $\mathrm{HNC}$, and the $\mathrm{HNC} / \mathrm{H} 2$ results for the pair distribution function.

\section{IV.2 The spinodal line}

In this subsection we study a pair potential with a minimum In particular we chose the LennardJones potential

$$
\phi(r)=4 \epsilon\left[\left(\frac{\sigma}{r}\right)^{12}-\left(\frac{\sigma}{r}\right)^{6}\right],
$$

where $\epsilon$ and $\sigma$ are positive parameters. The critical point for this fluid is at [27] $T_{c}^{*}=1.3120 \pm$ $0.0007, \rho_{c}^{*}=0.316 \pm 0.001$, and $P_{c}^{*}=0.1279 \pm 0.0006$.

Integral equations usually fail to have a solution at low temperature and intermediate densities, i.e. in the two-phases unstable region of the phase diagram. In particular it is well known that the HNC approximation is unable to reproduce the spinodal line, the locus of points of infinite compressibility in the phase diagram [12]. This is due to the loss of solution as one approaches the unstable region on an isotherm from high or from low densities. The line of loss of solution, in the phase diagram, is called termination line. According to the discussion of section II, the loss of solution for the HNC approximation can be traced back to the loss of strict convexity of the generating functional [28]. Indeed, using HNC approximation, we computed the bulk modulus from the compressibility equation $B_{c}$, on several isotherms as a function of the density. At low temperatures we found that both at high density and at low density we were unable to continue the isotherm at low values of $B_{c}$. Zerah's algorithm either could not get to convergence or it would converge at a non physical solution (with a pole in the structure factor at some finite wavevector $k$ ). Since HNC/H2 has, by construction, an always strictly convex generating functional, we expect it to be able to approximate a spinodal line (there should be no termination line).

In Figure 3 we show the behavior of $B_{c}$ on several isotherms as a function of density, calculated with the $\mathrm{HNC} / \mathrm{H} 2$ approximation. We see that now there are no termination points. $B_{c}$ never becomes exactly zero and the low temperature isotherms develop a bump in the intermediate density region. The same plot for the bulk modulus calculated from the virial pressure $B_{p}$, shows that at low temperatures this bulk modulus indeed becomes zero along the isotherms both at high and low densities.

In figure 4 the pressure is plotted as a function of the density on several isotherms for the $\mathrm{HNC} / \mathrm{H} 2$ approximation. Apart from the fact that we find negative pressures, the isotherms have a van der Waals like behavior.

The graphical analysis of the pressure plotted as a function of the chemical potential shows that the coexistence of the two phases (points where the curve crosses itself) is possible and is lost between $T^{*}=1.1$ and $T^{*}=1.2$. There generally are two points of coexistence. 


\section{Improving the closures}

The numerical results for $\mathrm{HNC} / \mathrm{H} 2$ exhibit interesting features as far as the coexistence region is concerned but show unambiguously a worst agreement with the MC structural data in correspondence with a marginal improvement in the thermodynamics.

We feel that the main problem is the difficulty of an accurate description of the bridge functions in terms of powers of the pair correlation function. Recent investigations on improved closures seem to point to the indirect correlation function $\gamma(r)$ or some renormalized version of it, as the best starting point for progress. However, before moving to more complex relations or functional dependences, we have explored two possible directions for improving the $\mathrm{HNC} / \mathrm{H} 2$ closure. In the first approach we have tried to follow the MHNC approach by Lado et al. [29]. In the second we have explored the possibilities of optimization offered by the numerical coefficient of the cubic term in the generating functional.

\section{V.1 Pseudo-bridge functions for $\mathrm{HNC} / \mathrm{H} 2$}

From the graphical analysis of the pair distribution function it is known [1] that $g(r)$ may be written as

$$
g(r)=\exp (-\beta \phi(r)+\gamma(r)+B(r)),
$$

where $\gamma(r)=h(r)-c(r)$ is the sum of all the series type diagrams and $B(r)$ the sum of bridge type diagrams. If we take

$$
B(r)=-\frac{1}{2} h^{2}(r)+G(r)
$$

we have that our HNC/H2 approximation amounts to setting $G(r)=0$. Rosenfeld and Ashcroft [6] proposed that $B(r)$ should be essentially the same for all potentials $\phi(r)$. We now make a similar proposal for the $G$ function and we will refer to it as pseudo bridge function. In the same spirit of the RHNC approximation of Lado [29] we will approximate $G(r)$ with the $G$ function of a short range (reference) potential $\phi_{0}(r)$. Assuming known the properties of the reference system, we can calculate the $G$ function as follows

$$
G_{0}(r)=\ln \left[g_{0}(r) e^{\beta \phi_{0}(r)}\right]-\gamma_{0}(r)+\frac{1}{2} h_{0}^{2}(r)
$$

The reference $\mathrm{HNC} / \mathrm{H} 2(\mathrm{RHNC} / \mathrm{H} 2)$ approximation is then

$$
g(r)=\exp \left(-\beta \phi(r)+\gamma(r)-\frac{1}{2} h^{2}(r)+G_{0}(r)\right) \quad .
$$

An expression for the free energy functional can be obtained turning on the potential $\phi(r)$ in two stages: first, from the noninteracting state to the reference potential $\phi_{0}(r)$ and then from there to the full potential $\phi(r)$. To this end we write

$$
\phi\left(r ; \lambda_{0}, \lambda_{1}\right)=\lambda_{0} \phi_{0}(r)+\lambda_{1} \Delta \phi(r),
$$


with $\Delta \phi(r)=\phi(r)-\phi_{0}(r)$. Following the same steps as in [30] we obtain for the excess free energy per particle

$$
f^{e x c}=f_{1}+f_{2}+f_{3}^{(0)}+\Delta f_{3}
$$

where the first two terms were already encountered in section II

$$
\begin{aligned}
& \beta f_{1}=\frac{1}{2} \rho \int d \mathbf{r}\left\{1+g(r)\left[\ln \left(g(r) e^{\beta \phi(r)}\right)-1\right]-h^{2}(r) / 2+h^{3}(r) / 6\right\} \\
& \beta f_{2}=\frac{1}{2 \rho} \int \frac{d \mathbf{k}}{(2 \pi)^{3}}\{\rho \hat{h}(k)-\ln [1+\rho \hat{h}(k)]\} .
\end{aligned}
$$

The third term is assumed known

$$
\beta f_{3}^{(0)}=-\frac{1}{2} \rho \int d \mathbf{r} \int_{0}^{1} d \lambda_{0} G\left(r ; \lambda_{0}, 0\right) \frac{\partial g\left(r ; \lambda_{0}, 0\right)}{\partial \lambda_{0}}=\beta\left(f^{(0)}-f_{1}^{(0)}-f_{2}^{(0)}\right)
$$

here $f^{(0)}$ is the excess free energy per particle of the reference system and $f_{1}^{(0)}, f_{2}^{(0)}$ are defined as in equations (5.7), (5.8) for the reference potential and its corresponding correlation functions. The last term is

$$
\beta \Delta f_{3}=-\frac{1}{2} \rho \int d \mathbf{r} \int_{0}^{1} d \lambda_{1} G\left(r ; 1, \lambda_{1}\right) \frac{\partial g\left(r ; 1, \lambda_{1}\right)}{\partial \lambda_{1}} .
$$

According to our proposal, $G$ is insensitive to a change in potential from $\phi_{0}$ to $\phi$. We may then approximate this last term as follows

$$
\beta \Delta f_{3} \approx-\frac{1}{2} \rho \int d \mathbf{r} G_{0}(r)\left[g(r)-g_{0}(r)\right]
$$

Now that we have the free energy we may consider it as a functional of both $h(r)$ and $G_{0}(r)$ and take its variation with respect to these functions. We find,

$$
\begin{aligned}
\beta \delta f^{e x c}= & \frac{1}{2} \rho \int d \mathbf{r}\left\{c(r)-h(r)+h^{2}(r) / 2+\ln \left[g(r) e^{\beta \phi(r)}\right]-G_{0}(r)\right\} \delta h(r) \\
& -\frac{1}{2} \rho \int d \mathbf{r}\left[g(r)-g_{0}(r)\right] \delta G_{0}(r) .
\end{aligned}
$$

It follows that the free energy is minimized when both the RHNC/H2 closure (equation (5.4)) is satisfied and when the following constraint

$$
\int d \mathbf{r}\left[g(r)-g_{0}(r)\right] \delta G_{0}(r)=0
$$

is fulfilled.

Taking the second functional derivative of $f^{e x c}$ with respect to $h(r)$ we find that also this free energy is a strictly convex functional of the total correlation function. This property was lacking in the RHNC theory and constitutes the main feature of the RHNC/H2 closure. As already stressed in section III.1 it ensures that if a solution to the integral equation exists it has to be unique. 
The constraint, as for RHNC, gives a certain thermodynamic consistency to the theory (see [30]). If we choose a hard sphere reference potential $\phi_{0}(r)=\phi_{0}(r ; \sigma)$ which depends on the length scale $\sigma$, the optimum values of the parameters that makes the generating functional a free energy can be determined by the constraint (5.13) which becomes

$$
\int d \mathbf{r}\left[g(r)-g_{0}(r)\right] \frac{\partial G_{0}(r)}{\partial \sigma}=0 \quad,
$$

However, neither the hard-sphere pseudo bridge functions nor some empirical attempt to model the unknown function via a Yukawa function provided useful results.

\section{V.2 Optimized HNC/H3 approximation}

For $\gamma=0 \mathrm{HNC} / \mathrm{H} 3$ reduces to $\mathrm{HNC} / \mathrm{H} 2$. For $\gamma>0$ the first peak of the pair distribution function is dumped respect to the one of the pair distribution function calculated with HNC/H2. For $\gamma<0$ the first peak increases giving in general a better fit to the simulation data.

In figure 5 we compare the pair distribution function of the Lennard-Jones fluid near its triple point, calculated with a molecular dynamic simulation [21], the HNC/H2 approximation, the approximation HNC/H3 with $\gamma=-0.203$ (at lower values of $\gamma$ Zerah' s algorithm would fail to converge), and the approximation $\mathrm{HNC} / \mathrm{H} 3$ with $\gamma=-0.1$ (when the generating functional of $\mathrm{HNC} / \mathrm{H} 3$ is still strictly convex). As we can see $\mathrm{HNC} / \mathrm{H} 3$ fits the simulation data better than $\mathrm{HNC} / \mathrm{H} 2$ even if the first peak is still slightly displaced to the left of the simulation data, a well known problem of the HNC approximation [6].

The best results are given by $\mathrm{HNC} / \mathrm{H} 3$ with $\gamma=-0.203$. Note that the $\mathrm{HNC} / \mathrm{H} 3$ generating functional at this value of $\gamma$ is not strictly convex (strict convexity is lost for $\gamma \lesssim-1 / 9$ ). The first peak of the static structure factor is at $k \sigma \simeq 6.75$ and has a magnitude of 2.41 , a quite low value for a liquid near the triple point. We have calculated the pressure and the internal energy. We found $\beta P / \rho \simeq 3.87$ and $U^{e x c} /(N \epsilon) \simeq-5.72$ (very close to the HNC results $\beta P / \rho \simeq 3.12$ and $\left.U^{e x c} /(N \epsilon) \simeq-5.87\right)$ to be compared with the simulation results [31] 0.36 and -6.12 respectively. The bulk moduli are $B_{c} \simeq 11.74$ and $B_{p} \simeq 36.61$ which shows that at the chosen value of $\gamma$ we do not have the thermodynamic consistency virial-compressibility and we do not improve on $\mathrm{HNC}$ inconsistency (using $\mathrm{HNC}$ we find $B_{c} \simeq 7.09$ and $B_{p} \simeq 32.72$ ).

\section{Conclusions}

In this paper we have analyzed the relations between generating functionals, thermodynamic consistency and uniqueness of the solution of the integral equations of liquid state theory. We think that the requirement of deriving from a free energy and the uniqueness of the solution are two important ingredients to enforce in the quest for better closures. The former requirement is of course crucial to get virial-energy consistency. But it is also important to get integral equations able to provide a closed formula for the chemical potential without additional approximations. This last issue looks highly desirable for applications of IET to the determination of phase diagrams. The latter is certainly a useful constraint from the numerical point of view but it is also a very strong condition, probably able to avoid some non physical behavior in the coexistence region, although this point would deserve further investigation. 
In this work, we have started an exploration of the capabilities of the combined requirement of consistency and uniqueness, starting with simple modifications to the HNC closure, corresponding to the addition of a square and a cubic power of $h(r)$ in the HNC functional. We found a couple of approximations ( $\mathrm{HNC} / \mathrm{H} 2$ and $\mathrm{HNC} / \mathrm{H} 3)$, which have built in the virial-free energy thermodynamic consistency and have a unique solution.

We numerically tested these closures on inverse power and the Lennard-Jones fluid. From the tests on the inverse power potential fluids one can see that the $\mathrm{HNC} / \mathrm{H} 2$ approximation is comparable to HNC for the thermodynamic quantities and performs worst than RY and even HNC for structural properties. The tests on the Lennard-Jones fluid revealed as this approximation does not suffer from the presence of a termination line (present in HNC and almost all the existing closures). This allowed us to follow isotherms from the low density to the high density region and this behavior would be very useful in the study of the phase coexistence. However, the thermodynamic results show only a marginal improvement on HNC and the structure is definitely worse.

Our trials to improve $\mathrm{HNC} / \mathrm{H} 2$ in the same spirit of the modified HNC approaches did not succeed. We feel that the main reason is in the difficulty of modeling the real bridge functions through a polynomial in the function $h(r)$. In this respect, approaches based on generating functionals depending on the indirect correlation function $\gamma(r)$ look more promising but we have not tried them yet.

Much better results for the structure are found with $\mathrm{HNC} / \mathrm{H} 3$ as is shown in figure 5 . However, probably for the same reasons just discussed, one has to renounce to have an approximation with a strictly convex generating functional depending on $h(r)$. The thermodynamics reproduced by $\mathrm{HNC} / \mathrm{H} 3$ is not yet satisfactory: due to the slight left shift of the main peak of the $g(r)$ the calculated pressure misses the simulation result. Nonetheless the presence of the free parameter $\gamma$ in HNC/H3 leaves open the possibility of imposing the thermodynamic consistency virial-compressibility. If the value of the parameter needed to have the consistency is bigger than $-1 /[3 \sup g(r)]$ then we would have an approximation which is completely thermodynamically consistent and have a unique solution. This strategy may eventually lead to discover that the price we have to pay to have a completely thermodynamically consistent approximation is the loss of strict convexity of the generating functional.

\section{A Appendix: Generating functionals of $\gamma$}

Often in the numerical solution of the $\mathrm{OZ}$ plus closure integral equation use is made of the auxiliary function $\gamma(r)=h(r)-c(r)$. Suppose that the closure relation can be written as

$$
\rho^{2} c(r)=-\Psi\{\gamma(r)\}
$$

where $\Psi$ is a local function of the function $\gamma$ and has a dependence on the value of the pair potential not explicitly shown.

We want to translate the integral equation into a variational principle involving functionals of $\gamma(r)$. Then we introduce a closure functional $\mathcal{F}_{c l}[\gamma]$ such that

$$
\frac{\delta \mathcal{F}_{c l}[\gamma]}{\delta \gamma(r)}=\Psi\{\gamma(r)\}
$$


and an OZ functional $\mathcal{F}_{O Z, c}[\gamma]$ such that, when $c(r)$ and $\gamma(r)$ satisfy the OZ equation, we have

$$
\frac{\delta \mathcal{F}_{O Z, c}[\gamma]}{\delta \gamma(r)}=\rho^{2} c(r)
$$

Then when both the closure and the OZ relations are satisfied, the functional $\mathcal{F}=\mathcal{F}_{c l}+\mathcal{F}_{O Z, c}$ is stationary with respect to variations of $\gamma(r)$, i.e.

$$
\frac{\delta \mathcal{F}[\gamma]}{\delta \gamma(r)}=0
$$

This is the variational principle sought.

Now, we want to find $\mathcal{F}_{O Z, c}$. The $\mathrm{OZ}$ equation in $k$ space is

$$
\rho \hat{c}^{2}(k)+\rho \hat{\gamma}(k) \hat{c}(k)-\hat{\gamma}(k)=0 \quad .
$$

When we solve it for $\hat{c}$ we find two solutions

$$
\hat{c}=\frac{-\hat{\Gamma} \pm \sqrt{\hat{\Gamma}^{2}+4 \hat{\Gamma}}}{2 \rho},
$$

where $\hat{\Gamma}(k)=\rho \hat{\gamma}(k)$ is always positive since

$$
\hat{\Gamma}=\rho^{2} \hat{h} \hat{c}=\rho^{2} \frac{\hat{h}^{2}}{1+\rho \hat{h}}=\rho^{2} \frac{\hat{h}^{2}}{S(k)},
$$

$S(k)$ being the liquid static structure factor which is positive definite for all $k$. Since $\hat{c}(k)$ is a function which oscillates around 0 , where $\hat{c}$ is negative we have to choose the solution with the minus sign, where it is positive the one with the plus sign. In particular, if the isothermal compressibility of the liquid $\chi_{T}$, is smaller than the one of the ideal gas $\chi_{T}^{0}$, we have that

$$
\hat{c}(0)=\frac{1}{\rho}\left(1-\frac{\chi_{T}^{0}}{\chi_{T}}\right)<0,
$$

and we have to start with the minus sign.

The functional we are looking for is then (see equation (30) in [15], with the constant set equal to zero)

$$
\mathcal{F}_{O Z, c}[\gamma]=\int_{0}^{1} d t \int d \mathbf{r} \gamma(r) \int \frac{d \mathbf{k}}{(2 \pi)^{3}} \frac{\rho}{2} e^{i \mathbf{k} \cdot \mathbf{r}}\left[-t \hat{\Gamma}(k)+s_{c}(k) \sqrt{t^{2} \hat{\Gamma}^{2}(k)+4 t \hat{\Gamma}(k)}\right],
$$

where $s_{c}(k)$ is +1 when $\hat{c}(k) \geq 0$ and -1 when $\hat{c}(k)<0$. Rearranging the integrals and making the change of variable $y=t \hat{\Gamma}$ we find

$$
\begin{aligned}
\mathcal{F}_{O Z, c}[\gamma]= & \frac{1}{2} \int \frac{d \mathbf{k}}{(2 \pi)^{3}} \int_{0}^{\hat{\Gamma}(k)} d y\left(-y+s_{c}(k) \sqrt{y^{2}+4 y}\right) \\
= & \int \frac{d \mathbf{k}}{(2 \pi)^{3}}\left\{-\hat{\Gamma}^{2} / 4+s_{c}(k)\left[(1+\hat{\Gamma} / 2) \sqrt{(1+\hat{\Gamma} / 2)^{2}-1}\right.\right. \\
& \left.\left.-\ln \left(1+\hat{\Gamma} / 2+\sqrt{(1+\hat{\Gamma} / 2)^{2}-1}\right)\right]\right\}
\end{aligned}
$$


If the closure relation has the form

$$
\rho^{2} h(r)=-\Psi\{\gamma(r)\},
$$

we can derive the corresponding functional using the same procedure. The final result is a functional $\mathcal{F}_{O Z, h}[\gamma]$ which differs from (A.10) for a plus sign in front of the first term in the integral.

However, by examining their second functional derivatives, we notice that both $\mathcal{F}_{O Z, c}[\gamma]$ and $\mathcal{F}_{O Z, h}[\gamma]$ are not certainly convex or concave. Thus, any check of the convexity properties of generating functionals of the $\gamma(r)$ function should be done on the full functional.

\section{B Appendix: Thermodynamic consistency}

For a homogeneous liquid interacting through a pair potential $\phi(r)$, the Helmholtz free energy per particle $f$ can be considered a functional of $\phi$. Indeed, in the canonical ensemble, one has

$$
\beta f[\phi]=\beta f_{0}-\frac{1}{N} \ln \left(\frac{1}{V^{N}} \int \exp \left[-\beta \frac{1}{2} \sum_{i \neq j} \phi\left(\mathbf{r}_{i j}\right)\right] d \mathbf{r}_{1} \cdots d \mathbf{r}_{N}\right),
$$

where $f_{0}$ is the free energy per particle of the ideal gas $(\phi=0)$ and $V$ is the volume of the liquid. Taking the functional derivative with respect to $\beta \phi(r)$ one finds

$$
\frac{\delta \beta f[\phi]}{\delta \beta \phi(r)}=\frac{\rho}{2} g(r)
$$

Imagine that we found a functional $\mathcal{A}([h],[\phi], \rho, \beta)$ that has an extremum for those correlation functions that solve the $\mathrm{OZ}$ and the closure system of equations. Suppose further that such functional has the following property

$$
\frac{\delta \beta \mathcal{A}}{\delta \beta \phi(r)}=\frac{\rho}{2} g(r)
$$

which can be rewritten more explicitly as follows

$$
\left.\frac{\delta \beta \mathcal{A}}{\delta \beta \phi(r)}\right|_{[h], \rho, \beta}+\left.\int d \mathbf{r}^{\prime} \frac{\delta \beta \mathcal{A}}{\delta h\left(r^{\prime}\right)}\right|_{[\phi], \rho, \beta} \frac{\delta h\left(r^{\prime}\right)}{\delta \beta \phi(r)}=\frac{\rho}{2} g(r) .
$$

Evaluating this expression on the correlation function $\bar{h}$ solution of the OZ plus closure system of equations, which is an extremum for $\mathcal{A}$, we find

$$
\left.\frac{\delta \beta \mathcal{A}}{\delta \beta \phi(r)}\right|_{[\bar{h}], \rho, \beta}=\frac{\rho}{2} \bar{g}(r)
$$

Then we can write

$$
\beta \mathcal{A}([\bar{h}],[\phi], \rho, \beta)=\left.\int d \mathbf{r} \frac{\delta \beta \mathcal{A}}{\delta \beta \phi(r)}\right|_{[\bar{h}], \rho, \beta} \beta \phi(r)+\mathcal{D}([\bar{h}], \rho, \beta),
$$


with $\mathcal{D}$ a functional independent of $\phi$. Changing variables to adimensional ones, $\mathbf{r}=\mathbf{r}^{*} \rho^{-1 / 3}$ and using equation (B.5) we find

$$
\beta \mathcal{A}\left(\left[\bar{h}^{*}\right],[\phi], \rho, \beta\right)=\frac{1}{2} \int d \mathbf{r}^{*} \bar{g}^{*}\left(r^{*}\right) \beta \phi\left(r^{*} \rho^{-1 / 3}\right)+\mathcal{D}\left(\left[\bar{h}^{*}\right], \rho, \beta\right)
$$

where we defined new distribution functions $g^{*}\left(r^{*}\right)=g\left(r^{*} \rho^{-1 / 3}\right)$. If $\mathcal{D}$ has no explicit dependence on $\rho$ then one readily finds

$$
\begin{aligned}
\rho \frac{\partial \beta \mathcal{A}\left(\left[\bar{h}^{*}\right],[\phi], \rho, \beta\right)}{\partial \rho} & =-\frac{\rho}{6} \int d \mathbf{r}^{*} \bar{g}^{*}\left(r^{*}\right) \beta \phi^{\prime}\left(r^{*} \rho^{-1 / 3}\right) r^{*} \rho^{-4 / 3} \\
& =-\frac{\rho}{6} \int d \mathbf{r} \bar{g}(r) \beta \phi^{\prime}(r) r \\
& =\beta P^{\text {exc }} / \rho,
\end{aligned}
$$

where again we used the fact that $\mathcal{A}$ has an extremum for $h=\bar{h}$. We used a prime to denote a derivative with respect to the argument and $P^{e x c}$ is the excess pressure of the liquid.

If $\mathcal{D}$ has no explicit dependence on $\beta$ we also find

$$
\begin{aligned}
\frac{\partial \beta \mathcal{A}\left(\left[\bar{h}^{*}\right],[\phi], \rho, \beta\right)}{\partial \beta} & =\frac{\rho}{2} \int d \mathbf{r} \bar{g}(r) \phi(r) \\
& =U^{e x c} / N
\end{aligned}
$$

where $U^{e x c}$ is the excess internal energy.

If $\mathcal{D}$ has no explicit dependence on both $\beta$ and $\rho, \mathcal{D}\left(\left[\bar{h}^{*}\right], \rho, \beta\right)=\mathcal{D}\left(\left[\bar{h}^{*}\right]\right)$, we conclude from equations (B.8) and (B.9) that

$$
\mathcal{A}\left(\left[\bar{h}^{*}\right],[\phi], \rho, \beta\right)=f^{e x c}(\rho, \beta)+\text { constant },
$$

where $f^{e x c}$ is the excess free energy per particle of the fluid. Under these circumstances we see from equation (B.8) that we have thermodynamic consistency between the route to the pressure going through the partial derivative of the free energy and the route to the pressure going through the virial theorem.

\section{Appendix: Strict convexity of $\mathcal{F}_{O Z}[h]$}

It can be proven that the functional

$$
\mathcal{F}_{O Z}[h]=\int \frac{d \mathbf{k}}{(2 \pi)^{3}}\{\rho \hat{h}(k)-\ln [1+\rho \hat{h}(k)]\},
$$

defined on the convex set

$$
D_{c}=\{h(r) \mid S(k)>0 \quad \forall k\} \quad,
$$

is a strictly convex functional. The strict convexity is a trivial consequence of the strict convexity of the integrand in equation (C.1). 
It remains to prove that $D_{c}$ is a convex set. Given two elements of this set $h^{\prime}$ and $h^{\prime \prime}$, we need to show that $h=\lambda h^{\prime}+(1-\lambda) h^{\prime \prime}$ is an element of $D_{c}$ for all $\lambda \in[0,1]$. Since

$$
\begin{aligned}
S(k) & =1+\rho \hat{h}(k) \\
& =1+\rho\left[\lambda \hat{h}^{\prime}(k)+(1-\lambda) \hat{h}^{\prime \prime}(k)\right] \\
& =1+\lambda\left[S^{\prime}(k)-1\right]+(1-\lambda)\left[S^{\prime \prime}(k)-1\right] \\
& =\lambda S^{\prime}(k)+(1-\lambda) S^{\prime \prime}(k)>0 \quad \forall \lambda \in[0,1],
\end{aligned}
$$

then $D_{c}$ is a convex set.

\section{References}

[1] J. P. Hansen and I. R. McDonald. "Theory of simple liquids". Academic Press, London, 2nd edition, 1986.

[2] C. Caccamo. Phys. Rep., 274:1, (1996).

[3] M. Born and H. S. Green. Proc. Roy. Soc., A188:10, (1946).

[4] J. K. Percus and G. J. Yevick. Phys. Rev., 110:1, (1958).

[5] J. M. J. van Leeuwen, J. Groenveld, and J. De Boer. Physica, 25:792, (1959).

[6] Y. Rosenfeld and N. W. Ashcroft. Phys. Rev. A, 20:1208, (1979).

[7] F. Lado, S. M. Foiles, and N. W. Ashcroft. Phys. Rev., A28:2374, (1983).

[8] L. Verlet. Mol. Phys., 41:183, (1980).

[9] G. A. Martynov and G. N. Sarkisov. Mol. Phys., 49:1495, (1983).

[10] D. -M. Duh and A. D. J. Haymet. J. Chem. Phys., 103:2625, (1995).

[11] T. Morita and K. Hiroike. Prog. Theor. Phys., 25:537, (1961).

[12] L. Belloni. J. Chem. Phys., 98:8080, (1993).

[13] P. D. Poll and N. W. Ashcroft. Phys. Rev., 35:5167, (1987).

[14] A. Schlijper, M. Telo de Gama, and P. Ferreira. J. Chem. Phys., 98:1534, (1989).

[15] W. Olivares and D. A. McQuarrie. J. Chem. Phys., 65:3604, (1976).

[16] S. Høye and G. Stell. J. Chem. Phys., 67:439, (1977).

[17] G. Pastore. Mol. Phys., 63:731, (1988).

[18] G. Pastore, O. Akinlade, F. Matthews, and Z. Badirkhan. Phys. Rev. E, 57:460, (1998).

[19] R. Kjellander, S. Sarman. J. Chem. Phys., 90:2768, (1989). 
[20] L. Lee. J. Chem. Phys., 97:8606, (1992).

[21] L. Verlet. Phys. Rev., 165:201, (1968).

[22] Our first trial should really be $c=-\ln g-\beta \phi$. Which should be called HNC/H1. We have tested numerically this closure and we found that it performed worst than $\mathrm{HNC} / \mathrm{H} 2$ both for the structure and for the thermodynamics of the system under exam.

[23] G. Zerah. J. Comp. Phys., 61:280, (1985).

[24] F. Lado. J. Comp. Phys., 8:417, (1971).

[25] F. J. Rogers and D. A. Young. Phys. Rev. A, 30:999, (1984).

[26] J. P. Hansen and D. Shiff. Mol. Phys., 25:1281, (1973).

[27] J. J. Potoff and A. Z. Panagiotopoulos. J. Chem. Phys., 109:10914, (1998).

[28] P. G. Ferreira, R. L. Carvalho, M. M. Telo de Gama, and A. G. Schlijper. J. Chem. Phys., 101:594, (1994).

[29] F. Lado. Phys. Rev. A, 8:2548, (1973).

[30] F. Lado, S. M. Foiles, and N. W. Ashcroft. Phys. Rev. A, 28:2374, (1983).

[31] L. Verlet. Phys. Rev., 159:98, (1967). 


\section{List of Tables}

i We compare various thermodynamic quantities as obtained from the RY, the $\mathrm{HNC}$, and the $\mathrm{HNC} / \mathrm{H} 2$ closure, for the inverse 12th-power fluid at the freezing point $(z=0.813)$. $U^{e x c} /(N \epsilon)$ is the excess internal energy per particle, $\beta P^{(v)} / \rho-$ 1 the excess virial pressure, $B_{c}$ and $B_{p}$ are the bulk moduli calculated from the compressibility and the virial equations respectively. . . . . . . . . .

ii We compare various thermodynamic quantities as obtained from the RY, the $\mathrm{HNC}$, and the $\mathrm{HNC} / \mathrm{H} 2$ closure, for the inverse 6th-power fluid at the freezing point $(z=1.54)$. $U^{e x c} /(N \epsilon)$ is the excess internal energy per particle, $\beta P^{(v)} / \rho-1$ the excess virial pressure, $B_{c}$ and $B_{p}$ are the bulk moduli calculated from the compressibility and the virial equations respectively. . . . . . . . .

iii We compare various thermodynamic quantities as obtained from the RY, the $\mathrm{HNC}$ and the $\mathrm{HNC} / \mathrm{H} 2$ closure, for the inverse 4th-power fluid at the freezing point $(z=3.92)$. $U^{e x c} /(N \epsilon)$ is the excess internal energy per particle, $\beta P^{(v)} / \rho-1$ the excess virial pressure, $B_{c}$ and $B_{p}$ are the bulk moduli calculated from the compressibility and the virial equations respectively. . . . . . . . . . . 21 


\begin{tabular}{|l|l|l|c|c|}
\hline \hline \multicolumn{1}{|c|}{ closure } & $U^{\text {exc }} /(N \epsilon)$ & $\beta P^{(v)} / \rho-1$ & $B_{c}$ & $B_{p}$ \\
\hline RY $(\alpha=0.603)$ & 2.626 & 18.359 & 69.782 & 70.125 \\
\hline HNC & 3.009 & 21.036 & 45.278 & 80.430 \\
\hline HNC $/ \mathrm{H} 2$ & 3.200 & 22.372 & 52.661 & 87.255 \\
\hline \hline
\end{tabular}

Table i: We compare various thermodynamic quantities as obtained from the RY, the HNC, and the $\mathrm{HNC} / \mathrm{H} 2$ closure, for the inverse 12th-power fluid at the freezing point $(z=0.813)$. $U^{e x c} /(N \epsilon)$ is the excess internal energy per particle, $\beta P^{(v)} / \rho-1$ the excess virial pressure, $B_{c}$ and $B_{p}$ are the bulk moduli calculated from the compressibility and the virial equations respectively. 


\begin{tabular}{|l|l|l|c|c|}
\hline \hline \multicolumn{1}{|c|}{ closure } & $U^{e x c} /(N \epsilon)$ & $\beta P^{(v)} / \rho-1$ & $B_{c}$ & $B_{p}$ \\
\hline $\mathrm{RY}(\alpha=1.209)$ & 4.114 & 39.027 & 110.952 & 111.420 \\
\hline $\mathrm{HNC}$ & 4.235 & 40.178 & 84.016 & 113.733 \\
\hline $\mathrm{HNC} / \mathrm{H} 2$ & 4.283 & 40.635 & 88.289 & 115.757 \\
\hline \hline
\end{tabular}

Table ii: We compare various thermodynamic quantities as obtained from the RY, the HNC, and the $\mathrm{HNC} / \mathrm{H} 2$ closure, for the inverse 6th-power fluid at the freezing point $(z=1.54)$. $U^{e x c} /(N \epsilon)$ is the excess internal energy per particle, $\beta P^{(v)} / \rho-1$ the excess virial pressure, $B_{c}$ and $B_{p}$ are the bulk moduli calculated from the compressibility and the virial equations respectively. 


\begin{tabular}{|l|l|l|c|c|}
\hline \hline \multicolumn{1}{|c|}{ closure } & $U^{e x c} /(N \epsilon)$ & $\beta P^{(v)} / \rho-1$ & $B_{c}$ & $B_{p}$ \\
\hline $\mathrm{RY}(\alpha=1.794)$ & 8.001 & 104.664 & 250.106 & 242.948 \\
\hline $\mathrm{HNC}$ & 8.047 & 105.277 & 223.328 & 244.212 \\
\hline $\mathrm{HNC} / \mathrm{H} 2$ & 8.068 & 105.542 & 226.966 & 257.678 \\
\hline \hline
\end{tabular}

Table iii: We compare various thermodynamic quantities as obtained from the RY, the HNC and the $\mathrm{HNC} / \mathrm{H} 2$ closure, for the inverse 4th-power fluid at the freezing point $(z=3.92)$. $U^{e x c} /(N \epsilon)$ is the excess internal energy per particle, $\beta P^{(v)} / \rho-1$ the excess virial pressure, $B_{c}$ and $B_{p}$ are the bulk moduli calculated from the compressibility and the virial equations respectively. 


\section{List of Figures}

1 Comparison of the Monte Carlo (MC), the HNC, and HNC/H2 results for the pair distribution function of the inverse 12th-power fluid at $z=0.813 . \quad$. . . . 23

2 Comparison of the Monte Carlo (MC), HNC, and $\mathrm{HNC} / \mathrm{H} 2$ results for the pair distribution function of the inverse 4 th-power fluid at $z=3.92 . \quad \ldots . . .24$

3 Behavior of $B_{c}$ of the Lennard-Jones fluid, on several isotherms as a function of the density for the $\mathrm{HNC} / \mathrm{H} 2$ approximation. . . . . . . . . . . . . . . 25

4 Behavior of the pressure of the Lennard-Jones fluid, on several isotherms as a function of the density for the $\mathrm{HNC} / \mathrm{H} 2$ approximation. . . . . . . . . . . . . 26

5 Comparison of the pair distribution function of a Lennard-Jones fluid at $\rho^{*}=$ 0.85 and $T^{*}=0.719$, computed from the molecular dynamic (MD) simulation of Verlet, the $\mathrm{HNC} / \mathrm{H} 2$ approximation, and the $\mathrm{HNC} / \mathrm{H} 3$ approximation. For $\mathrm{HNC} / \mathrm{H} 3$ we present results obtained setting $\gamma=-0.1$ (when the generating functional of the approximation is still strictly convex) and $\gamma=-0.203$ (which gives the best fit possible to the simulation data but does not ensure the strict convexity of the generating functional). . . . . . . . . . . . . 27 


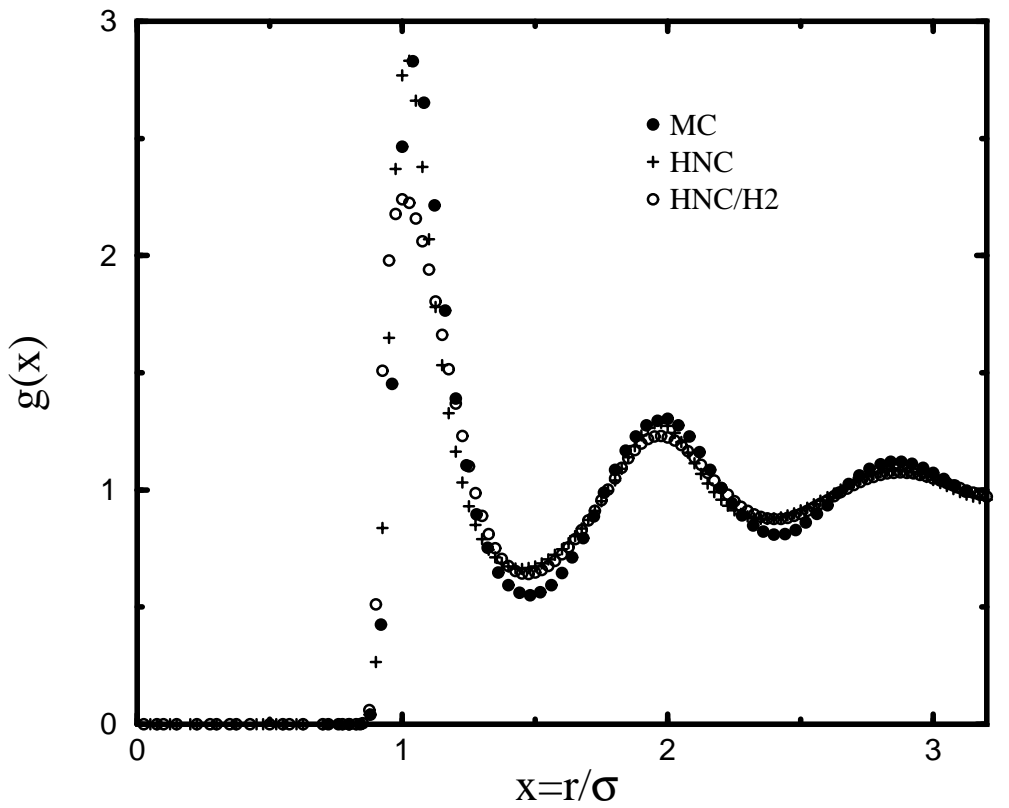

Figure 1: R. Fantoni and G. Pastore 


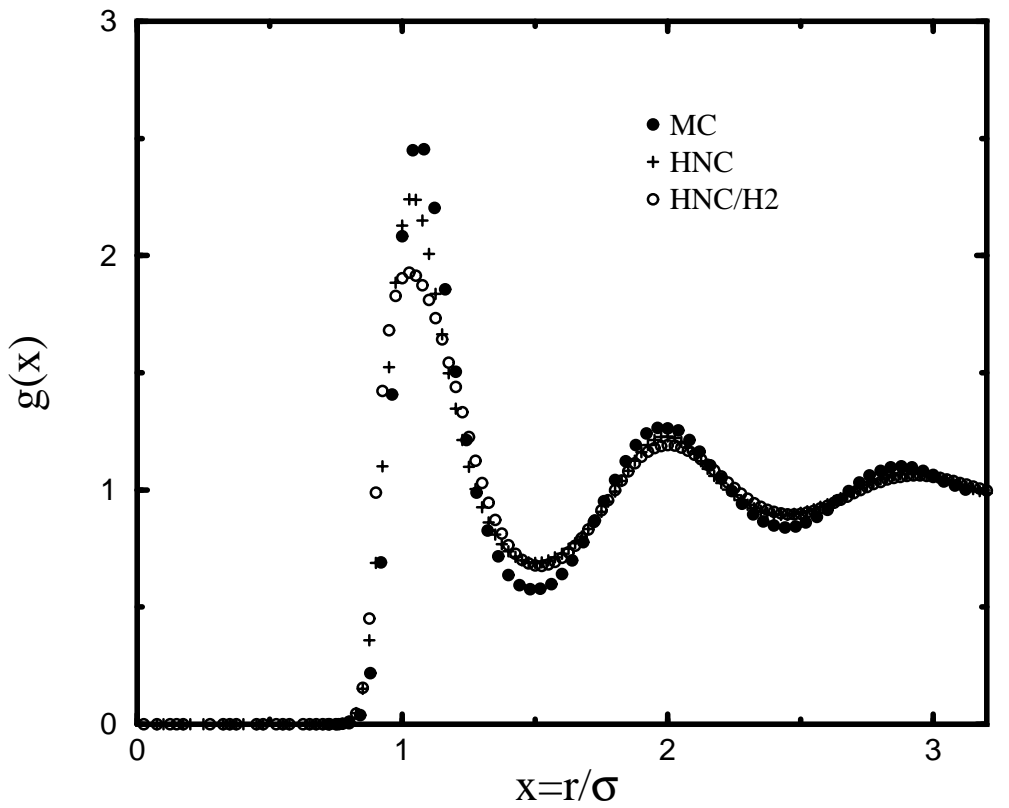

Figure 2: R. Fantoni and G. Pastore 


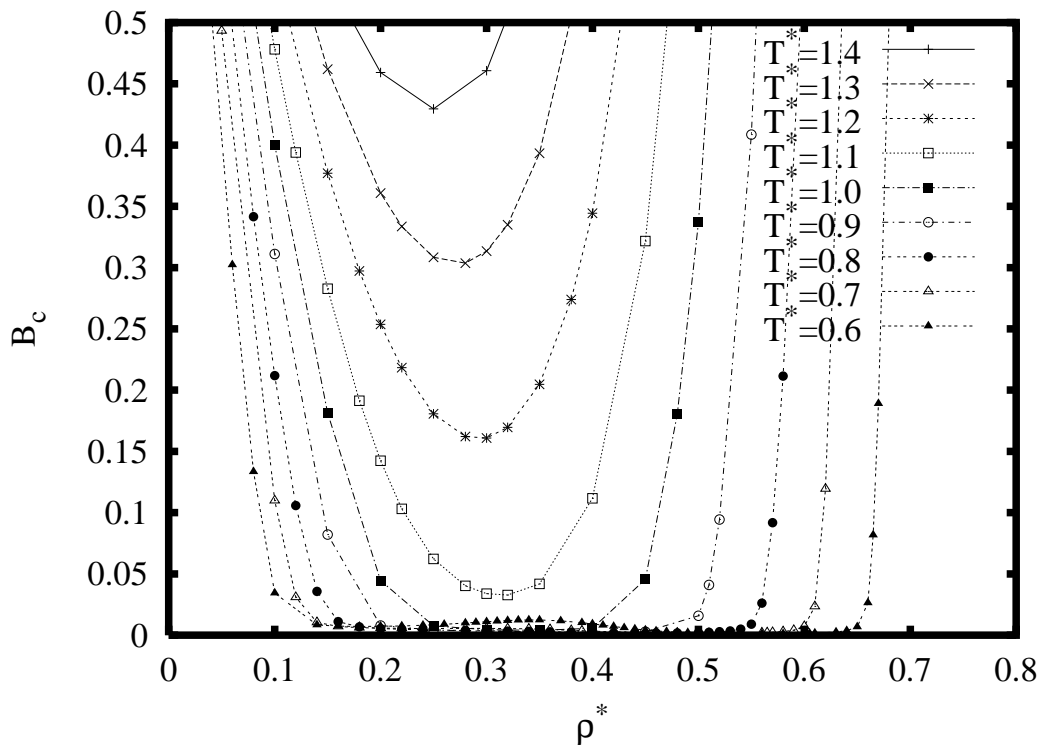

Figure 3: R. Fantoni and G. Pastore 


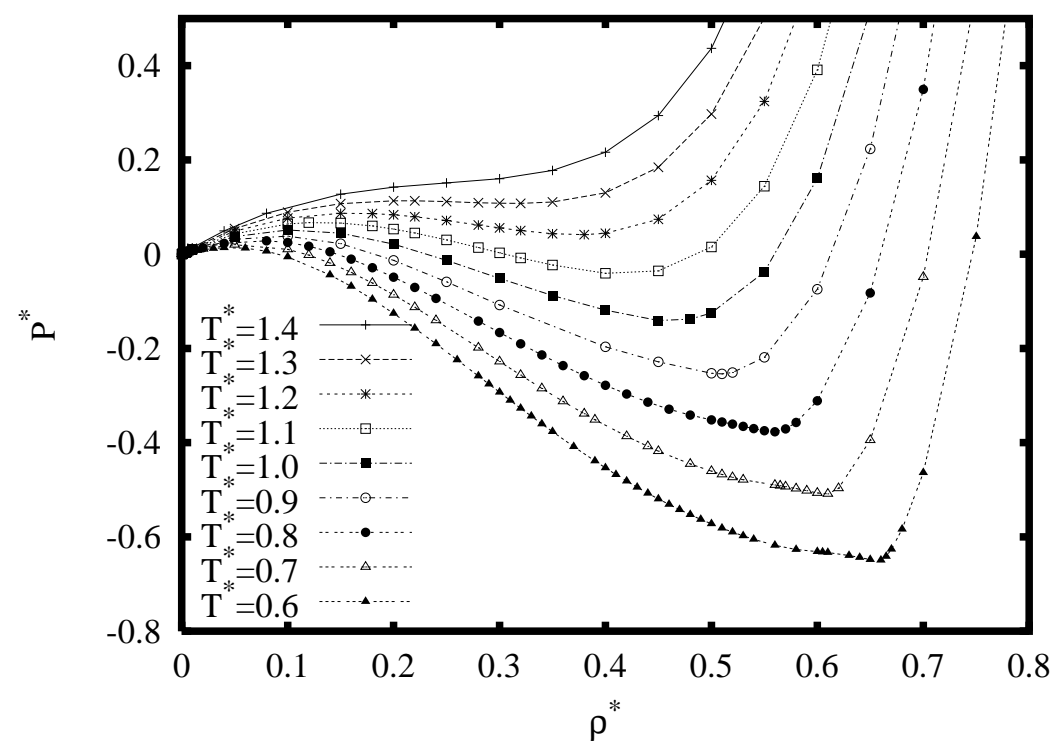

Figure 4: R. Fantoni and G. Pastore 


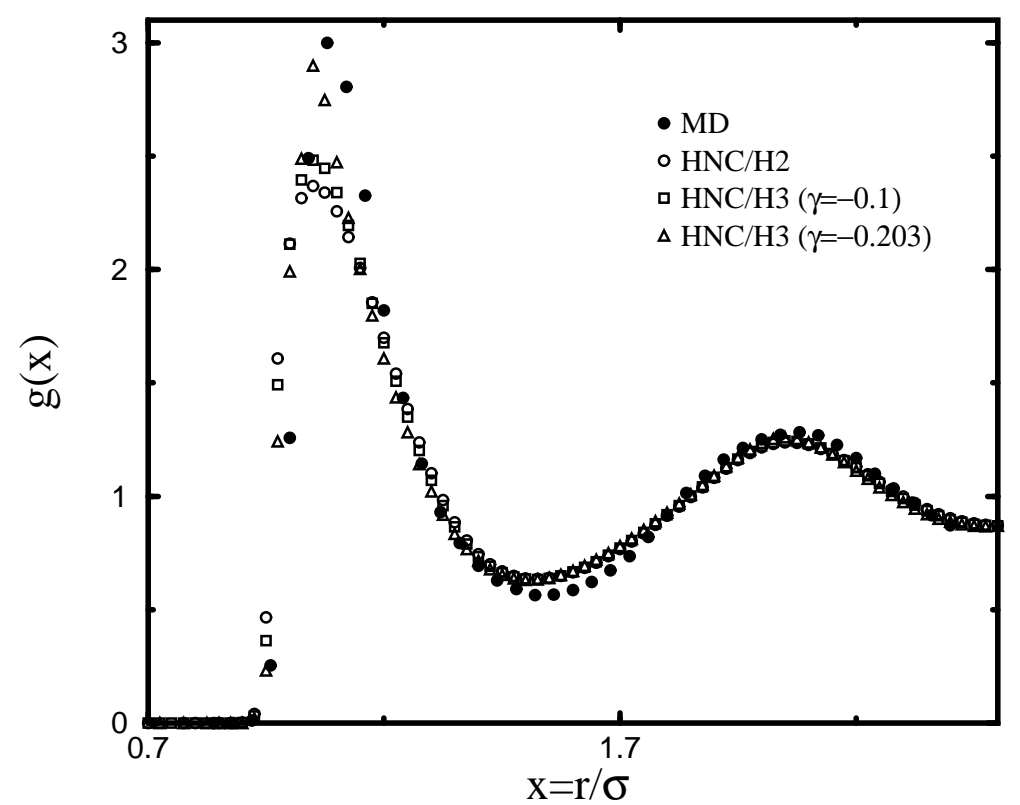

Figure 5: R. Fantoni and G. Pastore 